Draft chapter for Knowledge Cartography: Software Tools and Mapping Techniques. Eds. Okada, A., Buckingham Shum, S. and Sherborne, T. (Springer 2007)

\title{
Human-Agent Knowledge Cartography for e-Science: NASA Field Trials at the Mars Desert Research Station
}

\author{
Maarten Sierhuis ${ }^{1}$ and Simon Buckingham Shum ${ }^{2}$ \\ 1 Human-Centered Computing, NASA/Ames Research Center, Moffett Field, California, \\ 94035, USA, Maarten.Sierhuis-1@nasa.gov \\ 2 Knowledge Media Institute, The Open University, Milton Keynes, Buckinghamshire, \\ MK7 6AA, UK, sbs@acm.org
}

\begin{abstract}
This chapter describes the sociotechnical embedding of a knowledge cartography approach (Conversational Modelling) within a prototype e-science work system. This was evaluated over two 2-week field trials, simulating collaborative Mars-Earth geological exploration. We believe this work is the first demonstration of a knowledge mapping tool embedded within a human/software multiagent work system, with humans and agents reading and writing structures amenable to agent understanding and autonomous agent execution, and human understanding, annotation and argumentation. Secondly, in terms of the applied problem, we have demonstrated how human and agent plans, data, multimedia documents, metadata, discussions, interpretations and arguments can be mapped in an integrated manner, and successfully deployed in field trials which simulated aspects of mission workload pressure.
\end{abstract}

\section{Introduction}

At the time of writing, two NASA robotic rovers continue to explore the surface of Mars, over three years after landing in January 2004. While this and other missions astound us by what is possible with machine space exploration, there is much work already under way for human exploration. NASA is now planning to return to the moon, as the first step towards human exploration of Mars, a goal shared by the European Space Agency's Aurora programme. The work we report is part of NASA's human-centered computing programme whose research is to inform the creation of an effective and sustainable e-science work system between scientists on Earth and their astronaut colleagues on space missions. While interplanetary collaborative working is an extreme challenge with some unique features, the lessons we are learning are relevant to other projects confronting the challenges of distributed team working on one planet, such as simply Earth-based.

Our objective in this chapter is to describe how a particular form of knowledge cartography, called Conversational Modelling, has been used in realistic analogue simulations of collaboration between scientists on Mars and Earth, referred to as Mars-Earth scientific collaboration. We describe how the technical platform for 
Conversational Modelling, the Compendium tool (see also Chapters N, N, N) was embedded within NASA's broader Mobile Agents e-science work system, including people, robots, and software agents (Clancey, et al., 2003; 2004; 2005). We are prototyping tools not only for information sharing, but also for key sensemaking activities in which the information is interpreted and reified in forms suitable for communication and interpretation by human colleagues and software agents.

To set the context, we first introduce Compendium as a knowledge cartography research platform, architected to support interoperability with other tools, and the Conversational Modelling approach. We then introduce the NASA Mobile Agents project that has been designing and testing a Mars-Earth scientific collaboration work system, and explain the workflow supported by Compendium and Conversational Modelling in the analogue Mars mission simulations conducted at the Mars Desert Research Station in Utah. Attention then turns to the different genres of knowledge maps that evolved to support this process, and various evaluation indices we can use to reflect on the impact of this work. We then draw together our conclusions to date, and future work. ${ }^{1}$

\section{Compendium}

\subsection{User interface}

A technical objective of this work was to engage the tools and methods in their current state of development in non-trivial field tests, and from an action research perspective. The primary tool we used was Compendium [Compendiuminstitute-org] since (a) it has been designed from the start as a sensemaking-support environment

\footnotetext{
${ }^{1}$ Interactive web exports of the maps in this chapter, plus other materials, are presented on the project website: www.kmi.open ac.uk/projects/coakting/nasa
} 
ad so in principle had the potential to support the mission, and (b) we had expert users who could support both the Mars crew (the Crew) and the remote science team on Earth (the RST). Providing Compendium as a sensemaking-support tool to expert users is core to the vision of integrated Mars-Earth knowledge management. Compendium is a hypermedia tool providing a virtual canvas (a "map") on which one can arrange and structure nodes (Figure 1). Nodes may be data in any medium. Nodes may also correspond to ideas (eg. open issues; scientific hypotheses; theories arguments; evidence; decisions) or entities in a domain being modelled (e.g. experiments; services; data; substances; devices). Nodes may simply be clustered spatially, or can be optionally linked using unclassified or classified arrows. Compendium provides, therefore, a visual environment for personal or group information management, scaling by embedding maps within maps.

Compendium maps are not flat drawings, but multi-dimensional views onto a relational database that can be rendered in multiple formats, and accessed directly by other services to read/write content. The hypertext "transclusion" feature (Nelson 1987) enables a given node to appear and be updated in multiple views, that is, be given one or more meaningful contexts where it plays a role; as a result of transclusion corrections or updates to a node are immediately updated in every context in which it appears.

Semantic richness (such as additional node typing or categorization) is added to nodes through user-definable tags (metadata keywords) assigned to any concept (node) in the database to show connections through membership in a common category. This form of open-ended classification has been popularised in recent years by social bookmarking 'folksonomic' websites. Tags serve to specialize a node type with as many facets as required for it to play multiple roles in different contexts. At the end of the session all of the nodes so marked can be harvested, using a simple search algorithm. In modelling, nodes sharing a tag are often tracked as a library of nodes stored for future reuse. Tags may reflect generic meeting processes (e.g. Action-Jane), or may be driven by an underlying methodology that Compendium is being used to support (e.g. Data-Provider). Alternatively, ad hoc tags can be created on the fly, to reflect the emergence of a new theme. In short, tags are used to define meaning of a node in different contexts. Both, people and software agent can assign and use tags, based on specific rules that are in affect in different contexts. 


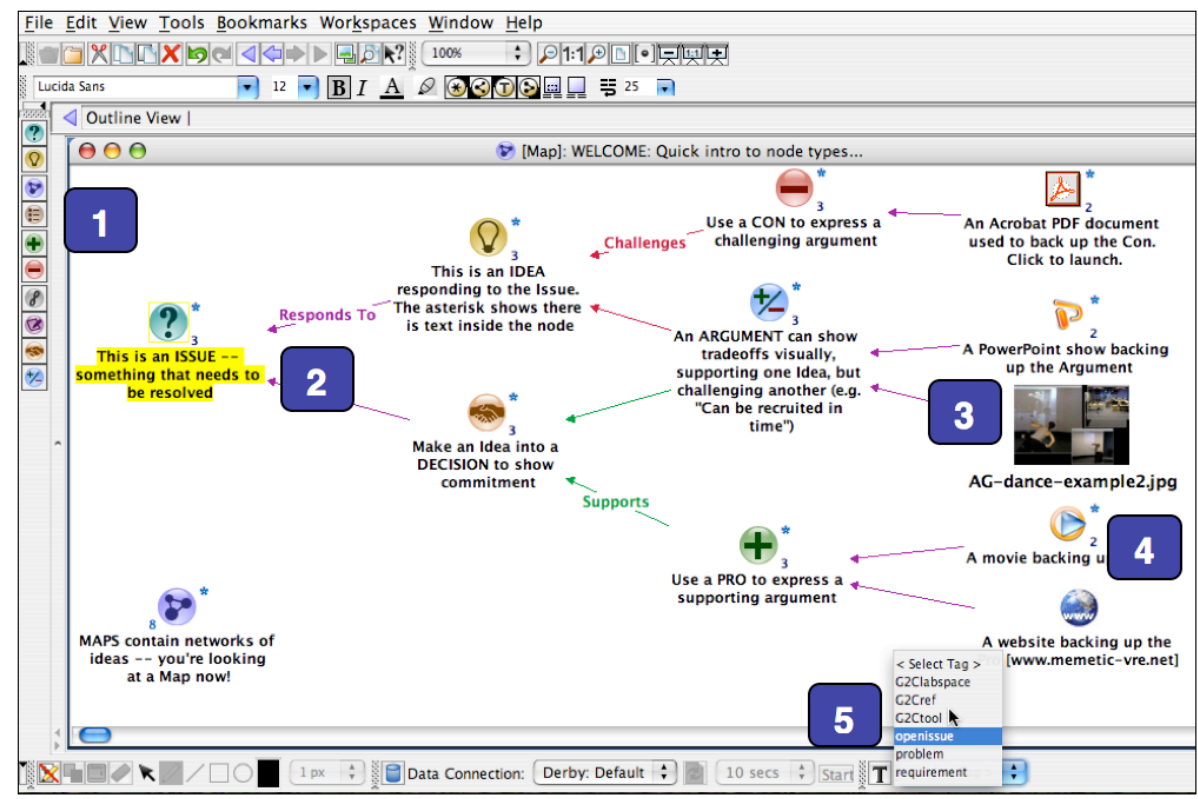

Figure 1: Compendium's visual language for IBIS, the Issue-Based Information System. Key to the numbered elements in the image: [1] Drag and drop nodes from the palette on the left of the screen onto the map. [2] Question-, light-bulb-, and handshake icons with (named) links in order to capture and link key issues, ideas, arguments and decisions. [3] Relevant media resources/websites can be linked into this discussion. (Users can also create their own palettes of icons.) [4] A digit superimposed on a node means that it appears in more than one map, i.e. the same idea or document can play roles in multiple contexts and conversations, yet be linked. [5] User-defined keyword tags can be annotated onto nodes to help when searching for related material across multiple maps

\subsection{IBIS-based Conversational Modelling}

Although it can be used for any kind of concept mapping, as shown in Figure 1, Compendium comes 'pre-loaded' with a visual language (icons, node types, and link types) for the Issue-Based Information System (IBIS) as proposed by Rittel ((Rittel 1972; Rittel et al. 1973) for tackling open-ended, ill-defined problems. IBIS provides a simple notation for connecting key issues, possible responses to these, and relevant arguments. Our previous work (Buckingham Shum et al. 2006) has de- 
scribed how we have evolved a set of practices for using Compendium and IBIS, which extends the use of IBIS from capturing a free-form discussion in real time (a skill termed Dialogue Mapping - (Conklin 2005)), to include more systematic domain modelling (termed Conversational Modelling - (Selvin 1999)).

A modelling approach focuses attention on a specific subset of issues and information, it may constrain the kinds of options one considers, and it may also focus attention on how one assesses them. A modelling approach also provides a syntactic and semantic framework (context) of rules that the users of the approach must obey in order for them to reach common understanding. This is useful for developing a sensemaking system for people, however, it is necessary if we want to create a sensemaking system for people interacting with software agents. This is, because software agents can only deal with concepts (nodes) if they have a formal meaning.

Our hypothesis at the start of the project was that to bridge the gap between people, liking to mix informal and formal sensemaking representations, and software agents, using a modelling tool that integrates both approaches is a possible solution for human-agent sensemaking interaction. Compendium was selected as the tool, because it provides both informal and formal representation capability.

In Compendium, a modelling approach is translated into a set of linked issue templates, which can also be created to deal with any well understood situation where there is a recommended approach to proceed (for instance, from best practice or a standard operating procedure). Compendium templates typically structure nodes with predefined tags, creating formal structure, and formal node metadata. Node and link labels may be left informal, intended only for human interpretation, or constrained in content for agent interpretation.

\subsection{A knowledge cartography research platform}

As an open research platform, with freely available source code, Compendium is distinctive from other tools in the effort that has been invested in designing for integration with the 'matrix' of other work system tools. Compendium is implemented as a cross-platform Java application that can swap between either the MySQL or Apache Derby relational databases. SQL and XML export/import assists data interoperability between clients and servers, and Semantic Web projects have added RDF compliant with different schemas (CoAKTinG, 2004; Memetic, 2006). Public Java application interface classes provide an interface for other systems to read and write to the database directly, so maps can be generated from another data source or interpreted for processing by another system. A shared MySQL database on a local area network supports rudimentary client-server architecture, but this is not optimised for internet access which can be slow. Data can be published to the Web as 
interactive image maps of concept/node networks or linear HTML outline documents (designed also to be accessible to screen-readers for visually impaired users). Web exports can be processed by extensions we have added to the open source Moodle e-learning content management system (OpenLearn, 2007).

Application-specific services (such as agent interoperability or map structure analysis) can be implemented over this substrate. For the Mobile Agents field trials, Compendium was linked into the Brahms multiagent infrastructure (Clancey, 1998; Sierhuis, 2001) by providing a Compendium software agent with access to read and write concept maps to the database. As illustrated below, issue templates-maps using predefined models of consistent concept network layout and tagging - could be interpreted by the software agents as Extra-Vehicle Activity plans ${ }^{2}$, and then populated by software agents or persons with captured science data over time, with metadata, as the science data was received from an EVA astronaut or from an EVA robotic assistant (i.e. a robot).

\section{Embedding Conversational Modelling in e-Science Workflow}

In a manned mission to Mars, the crew will necessarily be small, and must collaborate with scientists back on Earth, who themselves will work together in a distributed manner. NASA's Mars Exploration Rover mission ${ }^{3}$ has demonstrated that it is not practical to co-locate all the experts needed for multi-year missions. Designing collaboration support between the Crew and Remote Science Team (RST) raises

${ }^{2}$ Extra-Vehicle Activity is work performed by an astronaut outside the space craft.

${ }^{3}$ NASA's Mars Exploration Rover (MER) Mission is an ongoing unmanned Mars exploration mission, commenced in 2003, which sent two robotic rovers Spirit and Opportunity to explore the Martian surface and geology. 
some basic questions for designing computer-supported distributed cognition for science teams. The key challenge in this scenario is to manage, under continuous time pressure and with a high cost of errors, the gathering of science data and metadata, followed by its interpretation on both Mars and Earth, in order to inform scientific reasoning and decision-making for timely subsequent explorations. Furthermore, long time delays for communication between Mars and Earth make it impossible to have instantaneous communication between the two parties, making sharing of contextual (semantic) concept maps an ideal tool for collaboration over time and space. Although interplanetary collaboration has unique demands, the planning, collection and interpretation of information across time an space are tasks common to many Earth-bound e-science contexts, indeed, to knowledge-intensive work across all organisational sectors.

NASA's Mobile Agents Project is a multi-year, multi-research team project bringing together human-centered work systems design, multi-agent systems, speech dialogue, robotics, networking, semantic web, knowledge media. Throughout this process, the Mobile Agents Architecture (MAA) provides a means for modelling, simulating, implementing and managing a computer-supported Mars/Earth-based science work system. It is implemented in the Brahms and Java programming languages. Brahms is an agent-oriented language (Wooldridge 2002; Bordini et al. 2005) that provides a situated cognition perspective on the modelling of work practices (Clancey et al. 1998; Sierhuis 2001; Clancey et al. 2005; Sierhuis et al. 2005). Every team in the Mobile Agents project integrates their research software and hardware with the Brahms MAA. The MAA is a multi-agent workflow engine that connects all systems together and enables the deployment of a holistic exploration workflow system (Clancey et al. 2004)

The Mars Desert Research Station (MDRS) provides a mission testbed for identifying requirements, competitively testing alternative technologies/protocols, and training astronauts. Figure 2 shows photos of the MDRS 'Habitat', and astronauts on an EVA, gathering geological samples, and recording photos and voicenotes. 

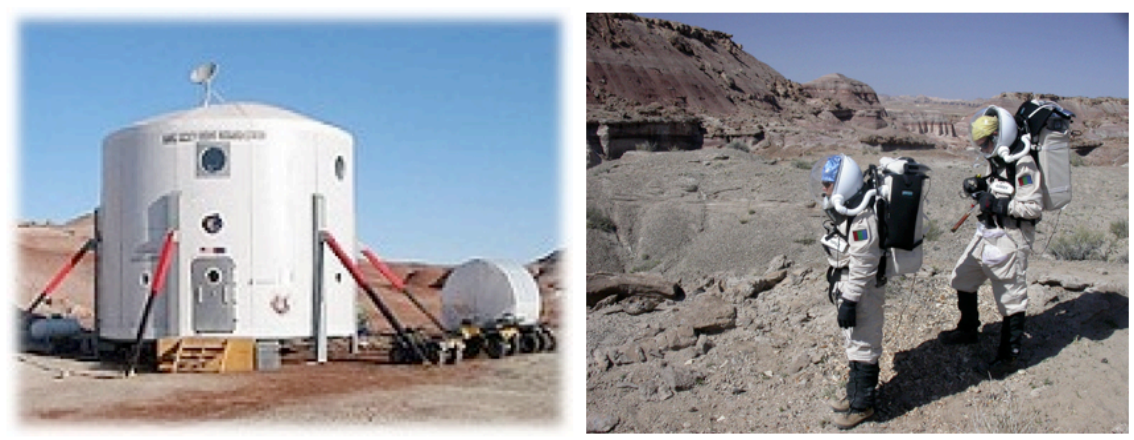

Figure 2: The Mars Desert Research Station Habitat ("the Hab") where the Crew lives, and the field geologist astronauts on an EVA to gather data.

The Mobile Agents 2004 field trial introduced a new research strand to MDRS analogue research, concerning collaboration with the RST who worked as a truly virtual team from offices and homes in California, New York state, and in two UK universities. Some members had never met physically (indeed, have yet to), simply being introduced by the project leader, and learning to work together via telephone, email, and shared documents via conventional office tools and a suite of collaboration tools. To explain the different roles played by the Compendium maps, it helps to have an overview of the workflow.

Figure 3 depicts the workflow of a typical EVA, explaining at what points Compendium is used:

1. The crew has a pre-EVA meeting in the habitat. This meeting is videotaped, and facilitated and captured via Compendium.

2. Both video and hypertext database is downlinked to Earth. A web-based MeetingReplay tool is automatically created from both the video and the Compendium database. The remote science teams (RST) watch the video on the web.

3. The RST have a teleconference facilitated in Compendium over the Web.

4. The RST's briefing is sent back to the crew in Compendium.

5. Based on the RST's Compendium map, the crew creates an EVA plan in Compendium.

6. When the crew is ready to start the EVA, the crew starts the Mobile Agents Architecture and asks the HabCom agent to load in the EVA plan directly from the Compendium database. 
7. The Plan Manager Assistant agent distributes the plan to all Personal Agents that manage communications on behalf of the two astronauts (Astro1, Astro2) and the EVA Robotic Assistant (ERA). The system is now ready to start the EVA.

8. During the EVA the ERA and astronauts perform the EVA plan and collecting mission data (geological photographs and voicenotes). This data flows via their Personal Agents back to the Habitat and are stored in Compendium (and another NASA database called ScienceOrganizer). Not shown in the figure is that email alerts are also sent to the RST notifying them of new data, and that the Compendium database is then mirrored on Earth for the RST.

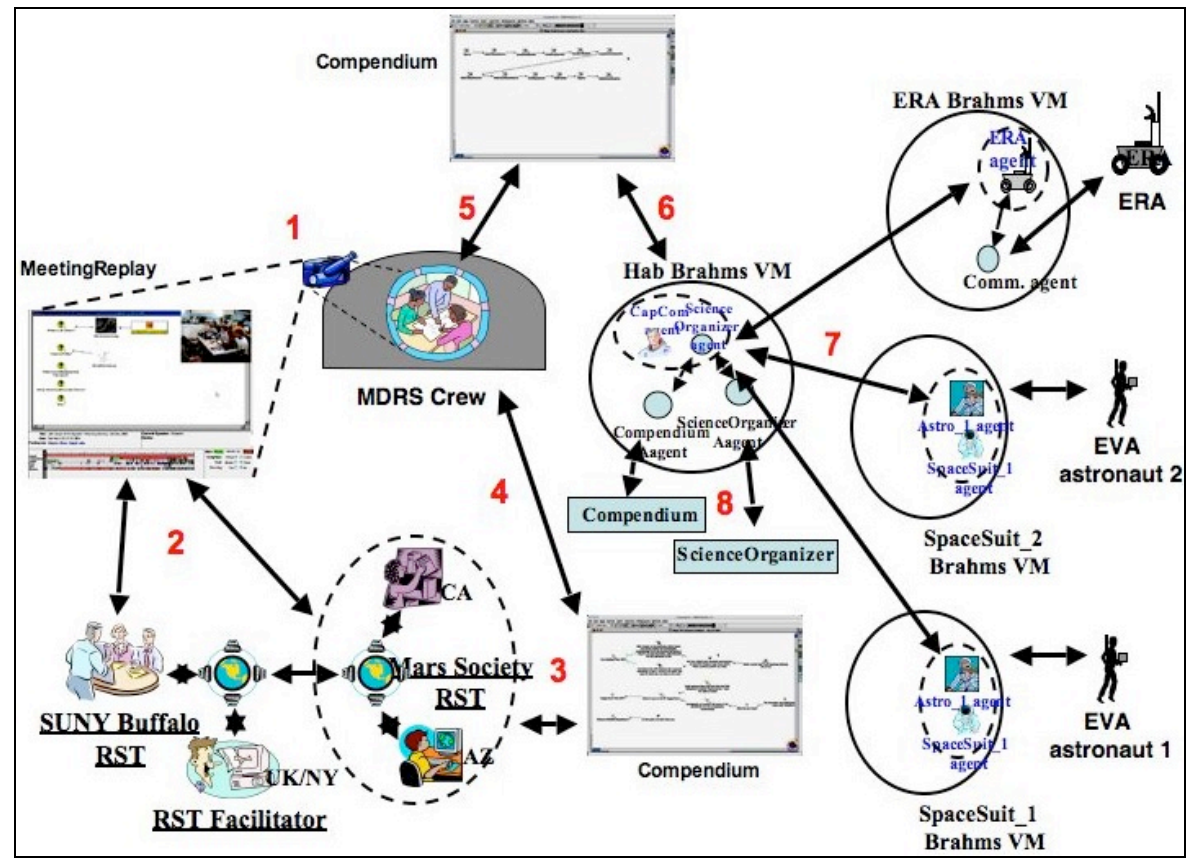

Figure 3. Workflow to plan, gather and analyse geological data in an EVA using the Mobile Agents architecture 
This chapter provides answers to a number of questions with respect to Compendium's representational expressiveness and usability:

- Can the RST and/or Crew specify plans in Compendium that can be read and executed by software agents?

- Can the scientists in the Crew communicate their daily plans for an EVA to the RST via Compendium?

- Can Compendium enable the RST to propose EVA plans for the Crew?

- In what ways can Compendium support post-EVA analysis of the collected science data?

- Will the RST be able to provide useful feedback to the Crew via Compendium in a form that can be absorbed in a timely manner?

In the two field trials, all RST teleconferences were audio and screen recorded, resulting in an archive of digital screen movies. All Crew meetings were video and screen recorded for integration within the Meeting Replay tool, and again, providing raw data for analysis. Table 1 below summarises the number of EVAs and different data types, to give an indication of the datasets that scientists in each two-week field trial generated and managed.

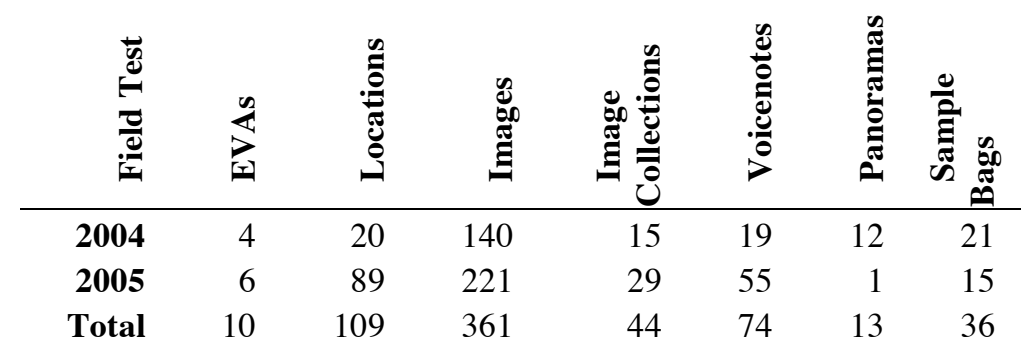

Table 1: EVAs and datasets from the 2004 and 2005 two-week field trials

To summarise, Compendium was used as both a personal and group knowledge mapping tool within the RST, between the RST and the Crew, and between the Crew and software agents supporting the planning and execution of EVAs.

\section{Genres of Compendium Map}

Mediating between the Crew and software agents. In the 2004 field trial, the Crew used a set of interlinked issue-templates to plan the route of the next EVA 
(Figure 4), constructing a visual map of the locations they wanted to visit and the activities to be conducted at each. These Compendium maps were then interpreted by the agents that coordinated commands and the flow of information during an EVA.

In the 2005 field trial, the RST took over the role of specifying the EVA plan. The RST worked through the templates, guided by their structure rather like completing a form, supported by the facilitator. However, an instance of the EVA plan template could be annotated using standard IBIS, e.g. with a rich description for the Crew of the activity to be undertaken, or to raise a query. The use of the EVA plan template enables the RST (or Crew) to formally communicate EVA plans for the software agent (formal in the sense that the EVA plan templates specifies the formal semantic of the EVA plan, using map-, question-, and position nodes using tags to specify EVA plan concept types). At the same time, the RST can use IBIS representations to communicate informally with the Crew (informal communication in the sense that Compendium does not force how to use IBIS formalisms).

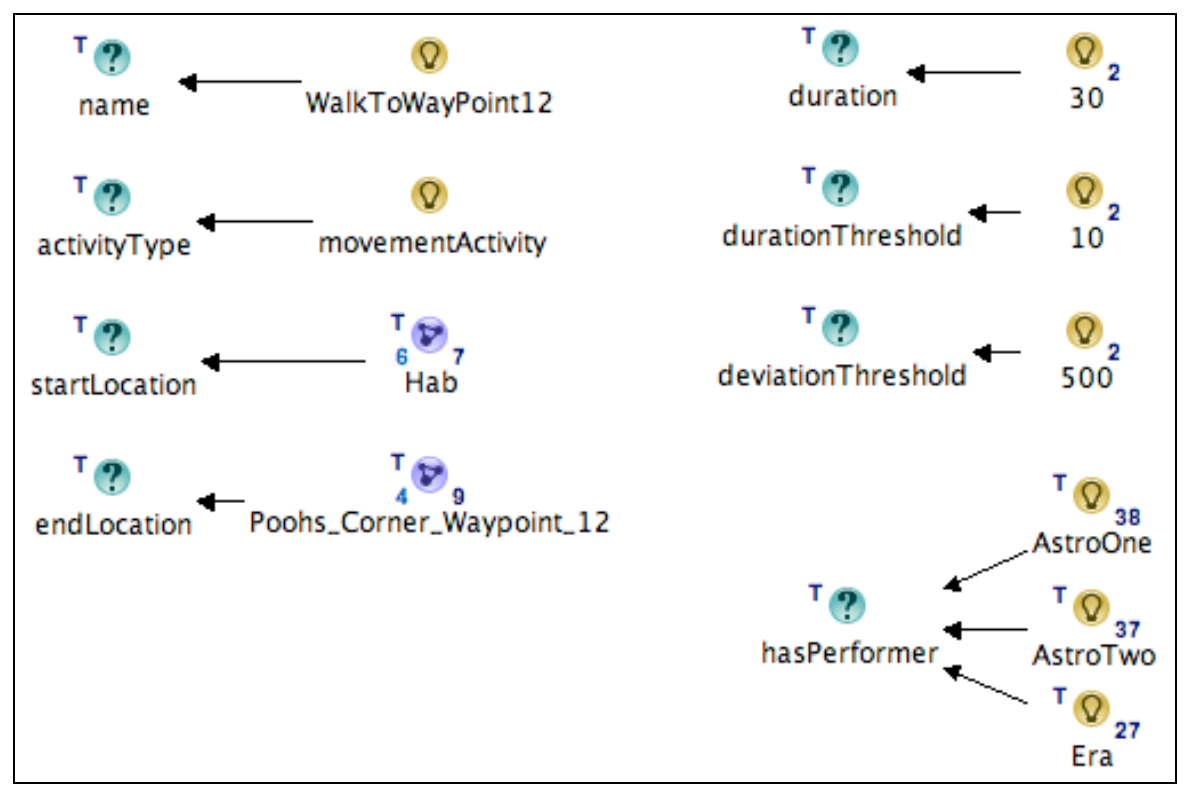

Figure 4. An EVA plan constructed by the Crew in Compendium using a Conversational Modelling template (in this example the EVA Activity template). This plan is read by the Brahms software agents that coordinated the EVA work flow. 
Viewing science data and metadata. All the data generated during an EVA (360 degree panoramic photos taken by robots, plus photographs and voice annotations recorded by astronauts) are stored in the Compendium database by a software agent, using specific predefined Compendium templates. Compendium renders the data and metadata as maps with nodes, links and tags (Figure 5), assisting the RST and/or Crew in seeing and navigating through the systematic use of tagging and transclusion. Thus, one could easily view all data from a given astronaut (tagged astrol), or all data associated with a particular work activity (a workactivity node transcluded by the software agent into multiple data maps).

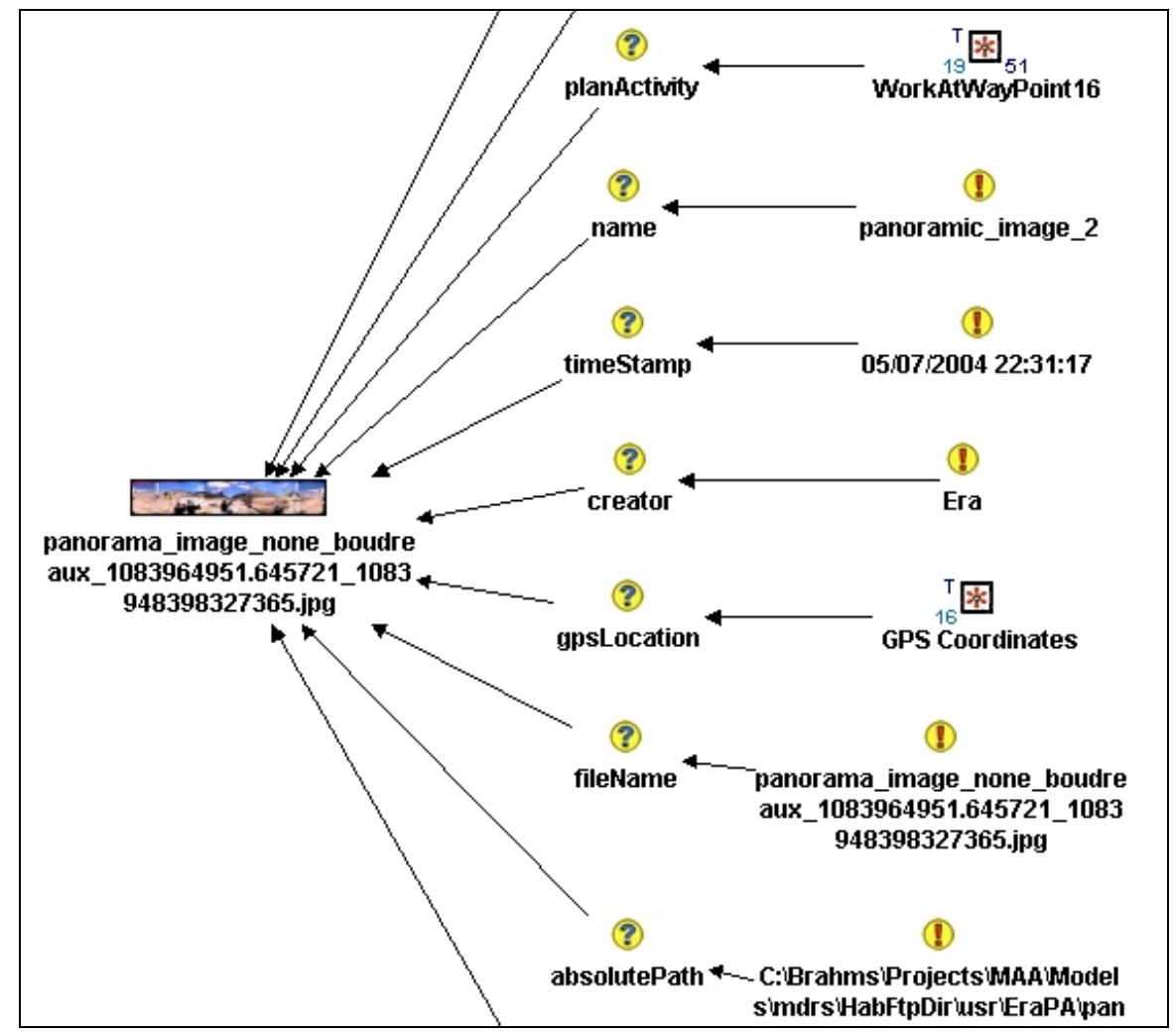

Figure 5. Following the EVA, a map is created and populated in Compendium. 
Crew and RST data analysis. As a team discussion unfolds, the contributions are simultaneously mapped on the screen (projected in the Hab in a crew meeting, or screen-shared over the internet during an RST teleconference using a desktop sharing tool). The Compendium Facilitator uses Dialogue Mapping to capture the team's discussions (e.g. Figure 6).

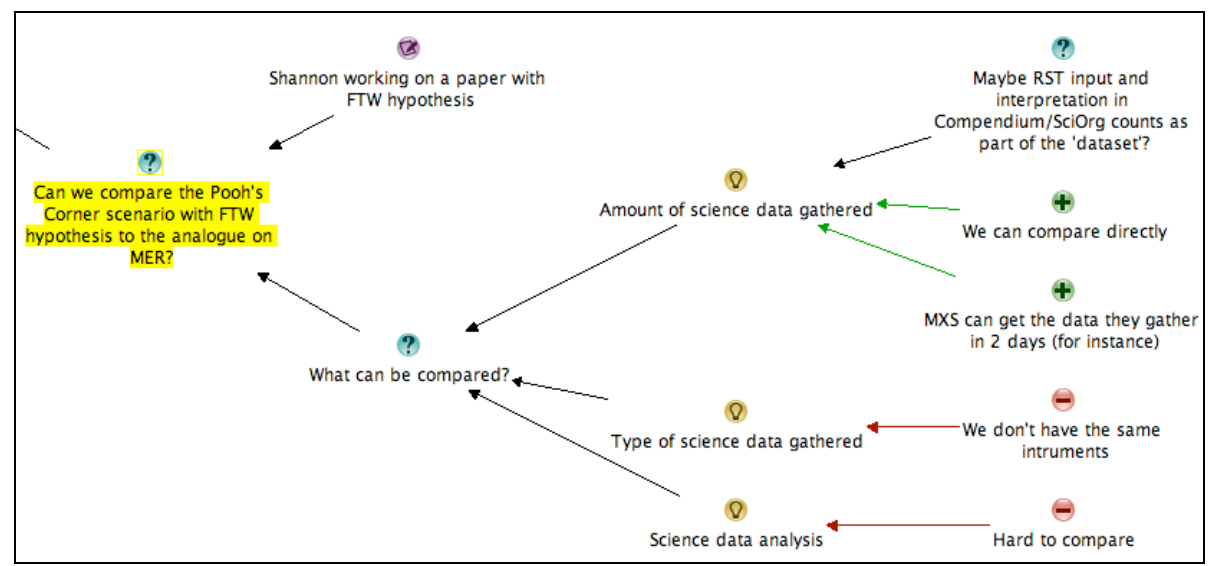

Figure 6: Dialogue Mapping RST deliberation over the issues, options and tradeoffs in a methodological discussion about evaluating the field trial.

Mediating RST feedback to the Crew. Prior to the 2004 field test, the RST defined a Crew Feedback template to organise feedback to the crew. This template allows for grouping ideas as Key Feedback and Suggestions (Figure 7). Every node is tagged accordingly, so that it is easy to find after a long ( $2+$ hour) meeting, and through Compendium's hypertext 'transclusion' mechanism, the connection can be preserved to the original Dialogue Map in which the feedback idea had arisen. This provided traceability of the ideas for both RST and the Crew. 


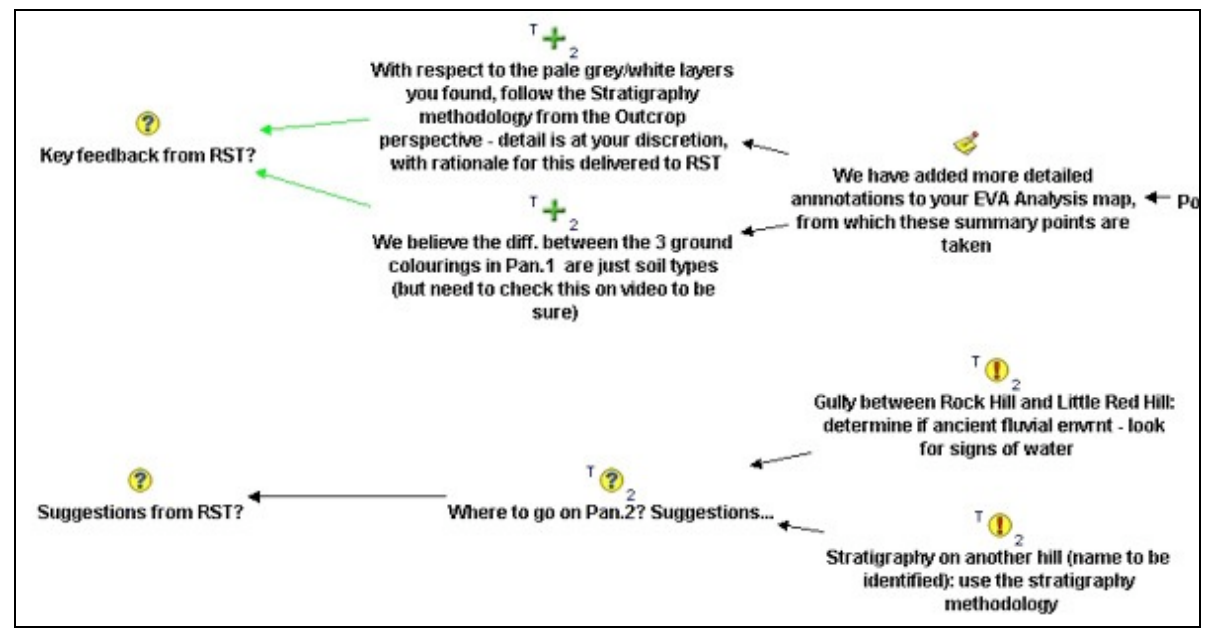

Figure 7. Compendium map summarizing the RST's feedback to the crew. Each node is hyperlinked to the detailed dialogue map in which it was created, enabling recovery of the original context in which that node was recorded.

Knowledge maps as indices into videos of Crew meetings. The communication delay between Mars and Earth makes synchronous conversation and the sharing of computer screens impossible. In collaboration with the University of Southampton, we developed a Meeting Replay tool, which combines meeting materials within an interface structured to enable quick and easy indexing for future navigation of the meeting record. During the mission we recorded the Crew's daily EVA planning meetings and delivering a replay of the meeting over the web to the RST, within a few hours. By experimenting with these techniques we hoped to see if the RST could gain a better understanding not only what a crew is deciding, but why, and how, in order to provide the best kind of feedback.

Figure 8 shows the web-based Meeting Replay tool. The upper region shows the video of the meeting and the Compendium map as the discussion progresses. The lower region contains summary information about the meeting - who was there, who was speaking, the agenda, and an overview of the current topic (derived from the Compendium map). Some of this information is presented as a timeline, providing a visual index for an RST member to navigate the video, jumping to relevant or interesting parts of the discussion by clicking on the timeline or moving the slider. 


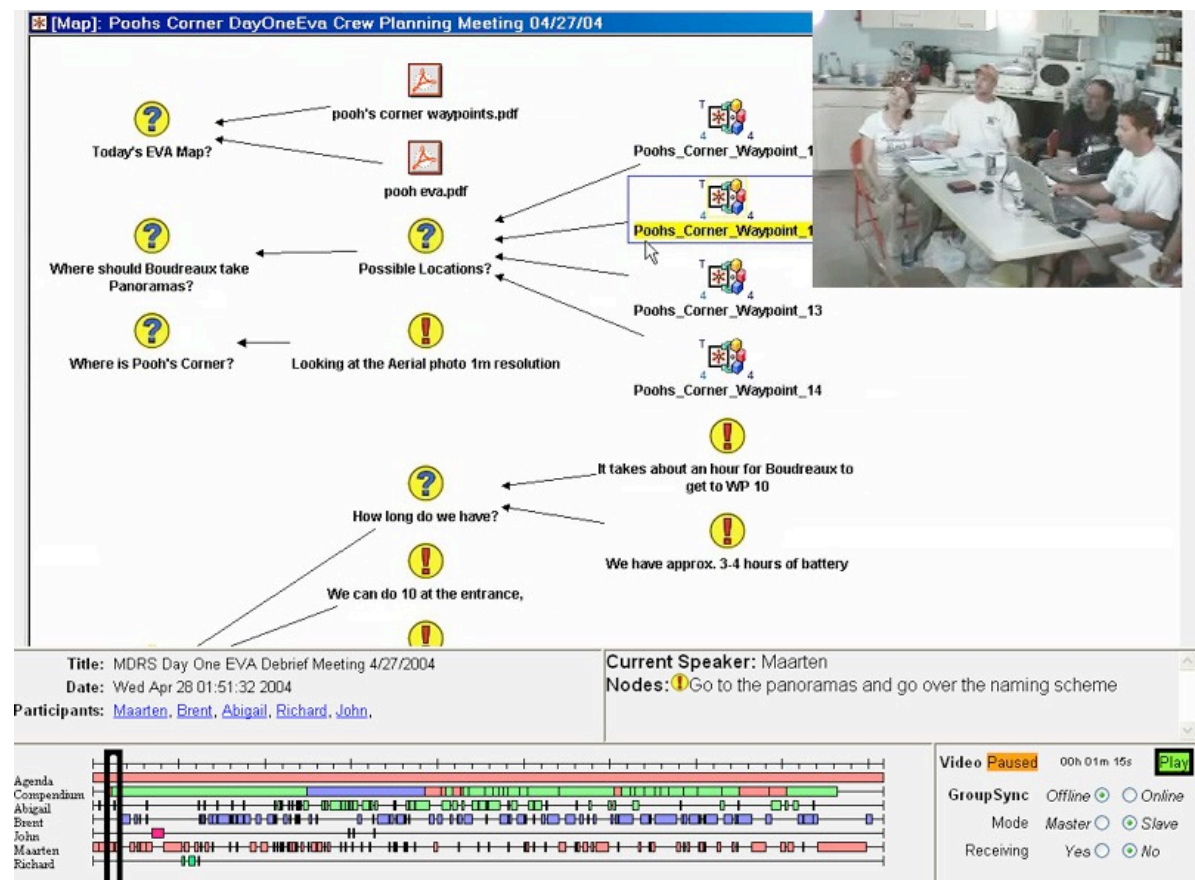

Figure 8. Web-based Meeting Replay tool. When reviewing the meeting replay, Compendium has been extended so that it can be used as a 'visual contents page' into the video. For instance, if the RST wants to see discussion prior to the recording of a particular decision, one can now click on this node in Compendium and the replay jumps to the point in the meeting where that node was recorded.

Communicating Crew analysis to RST. During the first field test in 2004, the Crew geologists used Compendium to send back to the RST collages of photographs linked to notes and questions (Figure 9). This proved to be an extremely productive way for the RST to understand how the Crew was thinking, as well as demonstrating the use of the tool in a way that the Crew geologists found intuitive. 


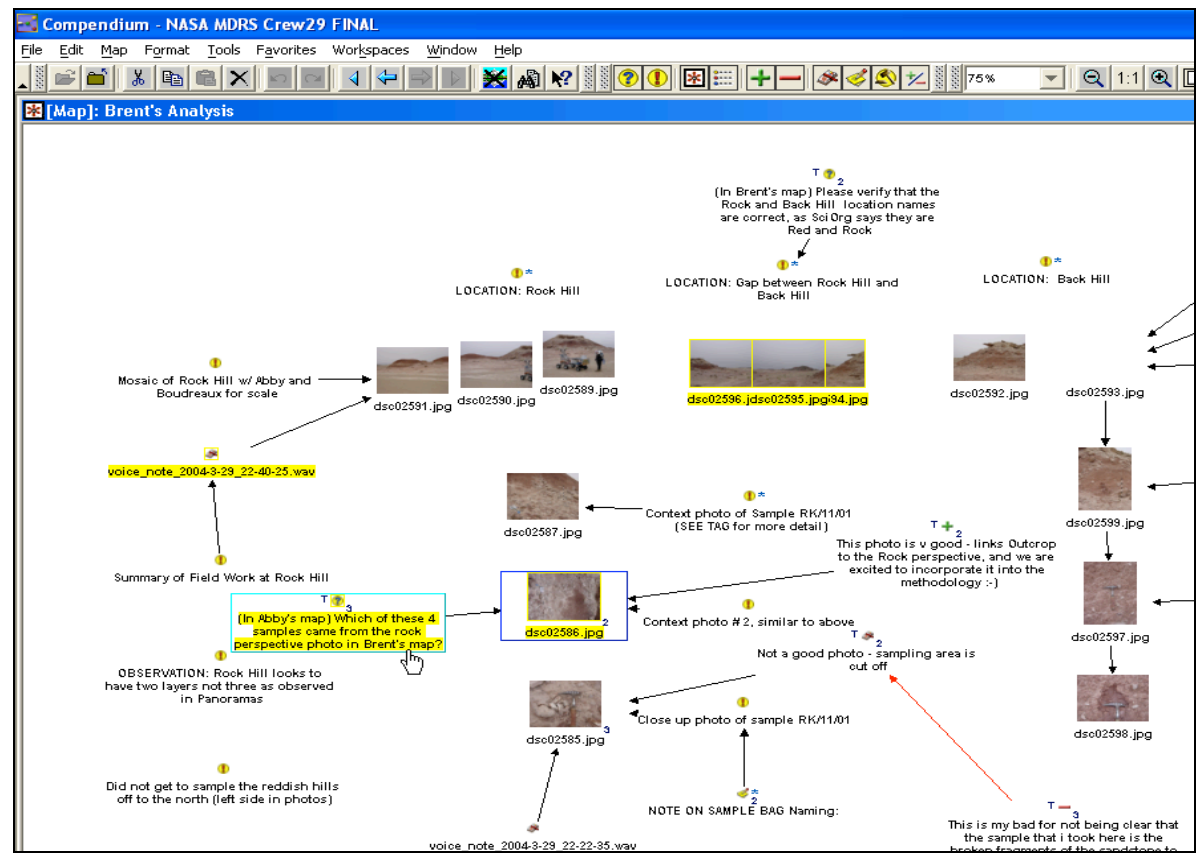

Figure 9: A Crew geologist arranges and annotates his photos on returning to the Hab after an EVA, which he then sends to the RST.

This map afforded the ability for the RST and Crew to exchange questions and answers, but via a medium in which the target data under discussion was always present (Figure 10). 


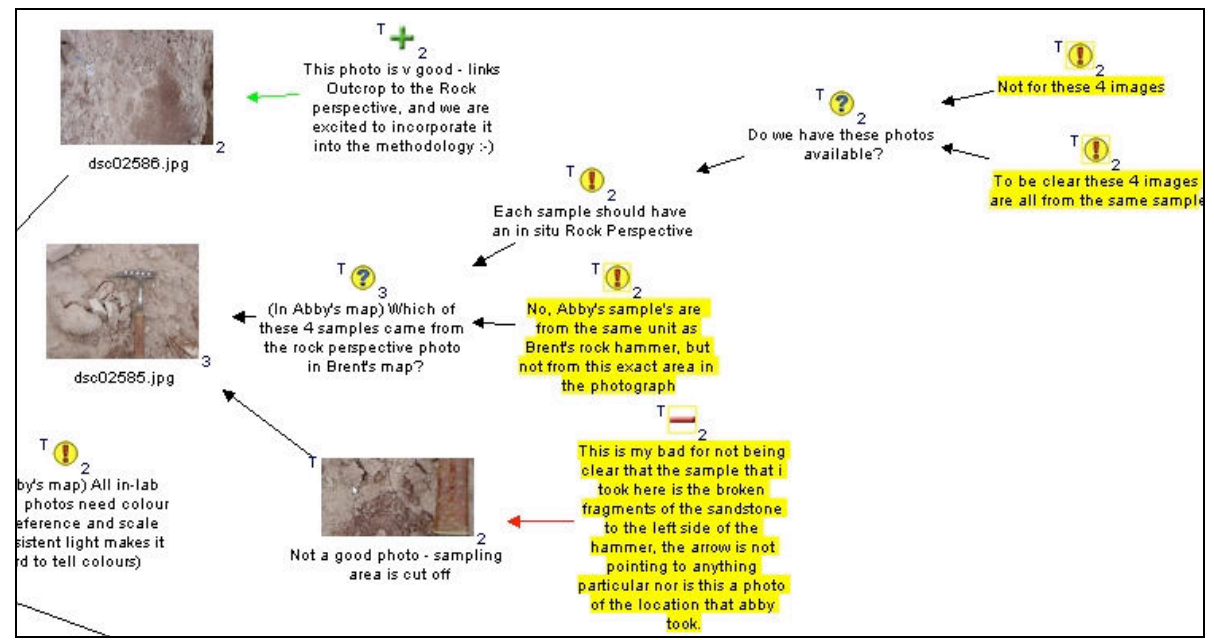

Figure 10: Crew-RST exchanges about photographic data. Using Compendium as a shared canvas for collaborative analysis between the scientists on 'Mars' and their remote support team on 'Earth'. First the scientists in the crew laid out photos of rock samples, and analysed them. The RST reviewed this and raised queries, linking them into the map, which accompanied another map containing their summary report. The crew then responded (yellow highlighted nodes).

RST Facilitator's web portal maps. A new genre of Compendium map emerged early in the 2004 field trial, whose use became standard practice for the remainder of that field trial and into 2005. To expedite the pace at which the RST could assimilate new data as they awoke in different time zones (sometimes in the early hours), the RST Facilitator performed the lengthy EVA data download and published this to the Web as interactive image maps. The RST members then had to simply visit the URL that was circulated by email. The map highlighted the elements needed to prepare for the meeting that would otherwise be embedded in multiple emails: briefing notes from the RST Leader, the links to WebEx and the Meeting Replay, and critically, the web export version of the Crew's Compendium maps.

Scaffolding scientific methodology. With its dialogue/argument mapping capability, Compendium presented the opportunity to explore a flexible knowledge management environment for agreeing on hypotheses, and considering how incoming data might be linked as evidence. The RST Facilitator helped the RST to set up new 
templates to manage the links between hypotheses and data. The Crew Facilitator worked with all the scientists to map the methodology that had been started by the RST in 2004, with the result that all scientists went into the 2005 trial with agreement and ownership of how they would operate in this respect (Figure 11).

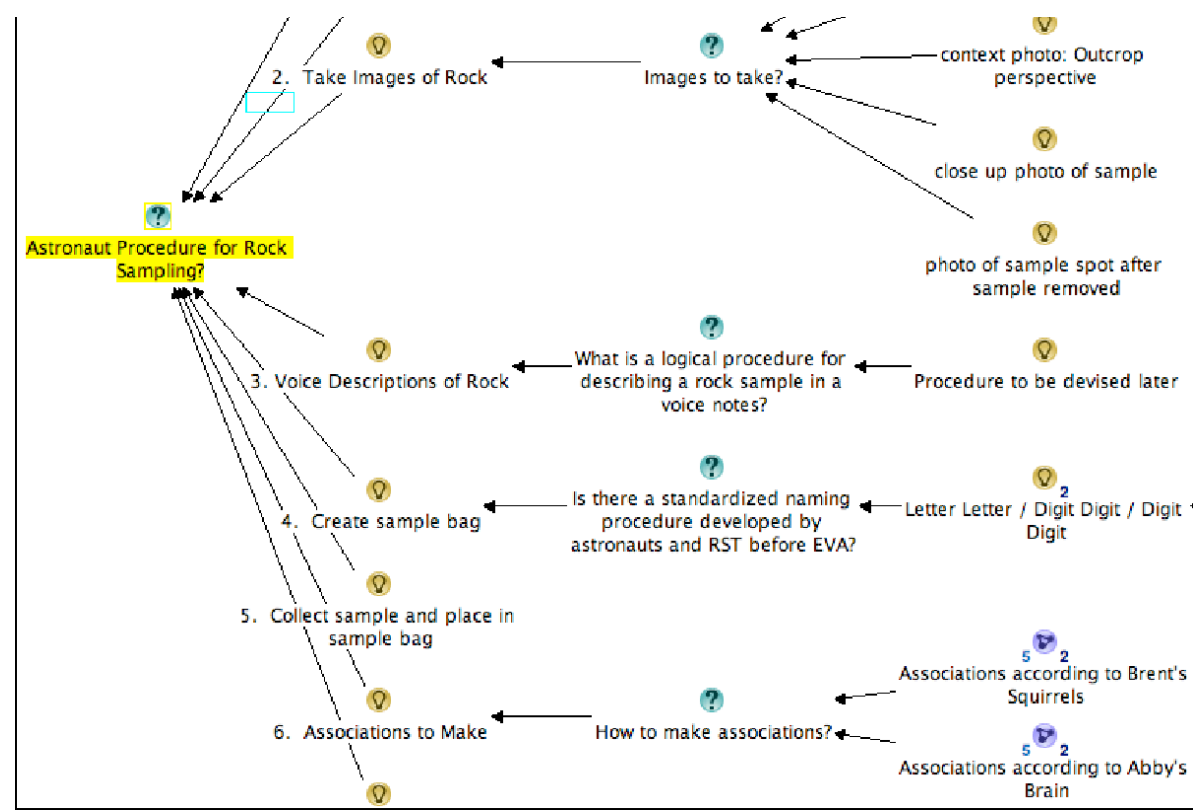

Figure 11: In MDRS 2005, the rock sampling methodology, which the RST started to map in the 2004 field trial, was mapped and agreed in the Hab by the Crew Facilitator and scientist astronauts. This proved invaluable when the Crew had to operate autonomously without RST input, yet were able to collect data that was coherent to the RST.

\section{Discussion and Future Work}

We propose that the work reported in this chapter makes contributions with respect to the state of the art in knowledge cartography software infrastructures, and with respect to addressing a realistic distributed sensemaking problem. Firstly, we believe this work is the first demonstration of a knowledge mapping tool embedded within a human/software multiagent work system. This was sucessful due to the respective 
software architectures of Compendium and the Mobile Agents systems. Together, these tools assisted the creation of Compendium agents that could read EVA planning maps designed by humans, and write science data maps for human annotation and argument, through the systematic use of IBIS-based modelling templates that constrained and scaffolded different genres of Crew and RST conversation.

This was exemplified by the Crew constructing science analysis maps in the same Compendium database as the original EVA Plan, and by the software agent automatically stored science data. By using copy and paste (the Compendium functions to create translusions of nodes), the Crew created the analysis maps (a new information context), using the previously captured images by the software agents. This functionality, indeed, created the hypothesized human-agent collaboration capability that was envisioned at the start of the project. Formal representations were used by software agents (the EVA Plans) to capture science data automatically (software agent created maps of images, etc), which in turn were later on used by people (the Crew or the RST) to create informal representations of the science data analysis. Then people could add questions and answers to the same maps, effectively creating a shared understanding of the data and the analysis. The Compendium tool provides all these capabilities within one environment. This fluid movement along the formality continuum is central to the success of this approach, enabling transformations in all directions between informal notes, semiformal Concept and Dialogue Maps, and formal template-driven maps. We must support not only formalization but also informalization.

Secondly, in terms of the applied problem, we have demonstrated how exploration plans, science data, metadata, multimedia documents, discussions, interpretations and arguments can be mapped in an integrated manner, as a component of the larger distributed Mobile Agents work system. This human-agent work system was tested in two 2-week field trials and succeeded in simulating aspects of true mission workload pressure. The field trials demonstrated the synchronous and asynchronous media affordances of Compendium in a multiplicity of roles:

- as a way to create formal information structures for understanding by software agents, from informal discussions by people.

- $\quad$ as a way to navigate richly linked data and metadata in maps written by software agents;

- $\quad$ as a real time sensemaking environment for co-located (Crew) meetings, and for online (RST) meetings;

- as an asynchronous medium for Crew-RST exchanges;

- $\quad$ as an asynchronous medium for scientists (Crew and RST) to program software agents when planning EVAs; 
- combining planned, formal modelling, with interpretive scientific and project management discourse which could move in unpredictable directions.

Although the Mobile Agents Architecture is a research architecture using custom NASA technologies in part, all other collaboration tools used the standard internet and a mix of commercial software products (e.g. Microsoft and Apple applications, and WebEx for screen-sharing over the internet, though there are free alternatives such as VNC), plus freely available tools, some of which are also open source (Brahms, Compendium; instant messengers). Technically, therefore, more broadly, other collaborative e-science projects and distributed teams could benefit from the collaborative knowledge cartography described here.

What about the skill set required to use Compendium fluently? We have highlighted the role played by the knowledge cartographer within the Crew and RST; both people and software agents can do the knowledge cartography. We have shown that untrained scientists can use Compendium to collage and annotate photos, and to develop diagrammatic templates to scaffold a methodology. Moreover, anyone fluent in switching between multiple applications could perform this role in meetings. Dialogue Mapping freeform discussions as IBIS structures, in real time, is one of the highest-level skills, but one that can be learnt (and taught: www cognexus.org). Such maps can be constructed post-hoc, from meeting notes, if it is too demanding in the meeting, The particular focus in this chapter on the use of specialised templates for Conversational Modelling (eg. for EVA Planning, or to structure the science data) demonstrates a hybrid approach, relieving the cognitive load on the dialogue mapper by scaffolding the discussion around a template 'agenda' of issues, driven by a modelling approach or metadata scheme.

This work is being developed in a number of directions. As Chapter X by Selvin demonstrates, we are seeking to articulate the nature of the knowledge cartography skill set as revealed through the analysis of session recordings. Compendium has established a significant user base $(\sim 10,000$ downloads of the tool, with $>500$ mailing list subscribers). Specifically, within NASA it continues to support the collaborative modelling of work systems (e.g. Sierhuis, 2006), while at the Open University, as a modelling tool providing visual templates for "Learning Design". On the technical front, we have integrated Compendium with the Access Grid, widely used in e-science/e-social science, to create a robust Meeting Replay environment (Buckingham Shum, et al., 2006). A Flash version of Compendium is being integrated into the Open University's FlashMeeting Web-videoconferencing tool (Scott, et al., 2007), and we are now investigating its integration as a visual environment for Web 2.0 applications such as blogging (e.g. Eisenstadt, 2006) and semantic, social bookmarking (OSC, 2007). Compendium is funded from public research grants in the UK and US, and our aim is to continue to offer it as a freely accessible, open, 
knowledge cartography research platform. We welcome your collaboration in taking it forward.

\section{Acknowledgments}

Large scale e-science of this sort takes a lot of effort from many people. This project was funded in part by a NASA Research Announcement (NRA) through the Information Systems' program Human-Centered Computing project, Mike Shafto selecting officer. This work was part of NASA Ames' Mobile Agent Project, with William J. Clancey as overall Principal Investigator and Maarten Sierhuis as overall Project Lead. Project members are civil servants and contractors at NASA-Ames in California and Johnson Space Center in Houston, TX. Satellite network services were funded by the NASA Research \& Education Network (NREN), and supported by researchers from NASA Glenn Research Center in Ohio. The United Kingdom's EPSRC funded the Advanced Knowledge Technologies consortium, of which the CoAKTinG Project is part: www.aktors.org/coakting. We are grateful to colleagues at the Open University's Knowledge Media Institute: Al Selvin for his contributions as an RST Facilitator and with assisting in our reflections on this role, and Michelle Bachler for programming Compendium. Our thanks to Kevin Page (University of Southampton) for support with the Meeting Replay tool. We are grateful to Shannon Rupert (MiraCosta College, now New Mexico State University), and the team at State University of New York at Buffalo: Stacy Sklar, Brent Garry, Abigail Semple, Melissa Farley, Brett Burkett, Kyle Fredrick, and Shannon Kobs. Our thanks also to the Northern California Mars Society mission support volunteers including especially Frank Crossman, as well the MDRS coordinator, Tony Muscatello, and the flight surgeon, Tam Czarnik, provided essential services during the two weeks at MDRS. Please see http://www.marssociety.org/MDRS/fso3/ (Crew 29: April 25May 8, 2004) and http://www.marssociety.org/MDRS/fs04/. (Crew 38: April 3-16, 2005 ) for the complete listing of mission participants, daily log entries and photographs.

\section{References}

Bordini, R.H., Dastani, M., Dix, J. and Seghrouchni, A.E.F., Eds. (2005). MultiAgent Programming: Languages, Platforms and Applications, Springer Science+Business Media, Inc. 
Buckingham Shum, S.J., Selvin, A.M., Sierhuis, M., Conklin, J., Haley, C.B. and Nuseibeh, B. (2006). Hypermedia Support for Argumentation-Based Rationale: 15 Years on from gIBIS and QOC. Rationale Management in Software Engineering. A. Dutoit, R. McCall, I. Mistrik and B. Paech. Berlin, Springer-Verlag: 111-132.

Buckingham Shum, S., Slack, R., Daw, M., Juby, B., Rowley, A., Bachler, M., Mancini , C., Michaelides, D., Procter, R., De Roure, D., Chown, T., and Hewitt, T. (2006). Memetic: An Infrastructure for Meeting Memory. Proc. 7th International Conference on the Design of Cooperative Systems, Carry-le-Rouet, France, 9-12 May. [PrePrint: www.memeticvre.net/publications/COOP2006_Memetic.pdf]

Clancey, W.J. (2001). Field Science Ethnography: Methods for Systematic Observation on an Expedition. Field Methods, 13 (3), p. 223-243.

Clancey, W.J. (2002). Simulating "Mars on Earth"-A Report from FMARS Phase 2. On to Mars: Colonizing a New World. F. Crossman and R. Zubrin, Apogee Books.

Clancey, W.J. (2004). Roles for Agent Assistants in Field Science: Understanding Personal Projects and Collaboration. IEEE Transactions on Systems, Man and Cybernetics-Part C: Applications and Reviews, 34 (2).

Clancey, W.J. (2006). Observation of Work Practices in Natural Settings. Cambridge Handbook on Expertise and Expert Performance. N.C. A. Ericsson, P. Veltovich, R. Hoffman.

Clancey, W.J., Sachs, P., Sierhuis, M. and van Hoof, R. (1998). Brahms: Simulating practice for work systems design. International Journal on Human-Computer Studies, 49, 831-865.

Clancey, W.J., Sierhuis, M., Alena, R., Berrios, D., Dowding, J., Graham, J.S., Tyree, K.S., Hirsh, R.L., Garry, W.B., Semple, A., Buckingham Shum, S.J., Shadbolt, N. and Rupert, S. (2005). Automating CapCom Using Mobile Agents and Robotic Assistants. American Institute of Aeronautics and Astronautics 1st Space Exploration Conference, Orlando, FL, Advanced Knowledge Technologies Project ePrint [htttp://eprints.aktors.org/375].

Clancey, W.J., Sierhuis, M., Alena, R., Crowford, S., Dowding, J., Graham, J., Kaskiris, C., Tyree, K.S. and Hoof, R.v. (2004). The Mobile Agents Integrated Field Test: Mars Dessert Research Station 2003. FLAIRS 2004, Miami Beach, Florida.

Clancey, W.J., Sierhuis, M., Damer, B. and Brodsky, B. (2005). The cognitive modelling of "day in the life" social behaviors using Brahms. Cognition and MultiAgent Interaction. R. Sun. New York, NY, Cambridge University Press: pp. 151184. 
CoAKTinG (2004): Collaborative Advanced Knowledge Technologies on the Grid. EPSRC/e-Science UK Project: www.aktors.org/coakting

Conklin, J. (2005). Dialogue Mapping: Building Shared Understanding of Wicked Problems. Chichester, Wiley.

Eisenstadt, M. (2004). More Dualling Blogs. Blog Entry, November 9th, 2004, Knowledge Media Institute, Open University, UK: http://kmi.open.ac.uk/people/marc/2004/11/09/more-duelling-blogs

Memetic (2006): Meeting Memory Technologies Informing Collaboration. JISC UK Project: www.memetic-vre.net

Nelson, T. (1987). Literary Machines (Ed. 93.1).

OpenLearn (2007). OpenLearn LabSpace, Open University, UK: http://labspace.open.ac.uk

OSC (2007): Open Sensemaking Communities project, Knowledge Media Institute, Open University, UK: http://kmi.open.ac.uk/projects/osc

Rittel, H.W.J. (1972). Second Generation Design Methods. Interview in: Design Methods Group 5th Anniversary Report: DMG Occasional Paper, 1, 5-10.

Rittel, H.W.J. and Webber, M.M. (1973). Dilemmas in a General Theory of Planning. Policy Sciences, 4, 155-169.

Scott, P., Tomadaki, E. and Quick, K. (2007). The Shape of Live Online Meetings. International Journal of Technology, Knowledge and Society.[PrePrint: http://kmi.open.ac.uk/projects/osc/docs/Shape $\% 20$ of\%20Live $\% 20$ Online $\% 20 \mathrm{Me}$ etings, \%20The.pdf]

Selvin, A. (1999). Supporting Collaborative Analysis and Design with Hypertext Functionality. Journal of Digital Information, 1 (4).

Sierhuis, M. (2001). Modelling and Simulating Work Practice; Brahms: A multiagent modelling and simulation language for work system analysis and design. Social Science Informatics (SWI). Amsterdam, The Netherlands, University of Amsterdam, SIKS Dissertation Series No. 2001-10: 350.

Sierhuis, M. (2006). Collaboratively Modeling Mission Control at NASA. Compendium Institute News, $11^{\text {th }}$ Mar, 2006.

http://news.kmi.open.ac.uk/rostra/news.php?r=55\&t=2\&id=20

Sierhuis, M., Clancey, W.J., Alena, R.L., Berrios, D., Shum, S.B., Dowding, J., Graham, J., Hoof, R.v., Kaskiris, C., Rupert, S. and Tyree, K.S. (2005). NASA's Mobile Agents Architecture: A Multi-Agent Workflow and Communication System for Planetary Exploration. $i$-SAIRAS 2005, München, Germany, European Space Agency.

Wooldridge, M. (2002). An Introduction to MultiAgent Systems, John Wiley \& Sons Ltd. 
Sierhuis and Buckingham Shum 